\title{
Programa para diagnóstico molecular de hemofilia A y B en Colombia
}

\author{
- Luz Karime Yunis Hazbun, Yolima Parrado Jara, Adriana Linares Ballesteros, Juan José Yunis Londoño
}

Universidad Nacional de Colombia (Bogotá, D.C.)

Introducción: desarrollar un programa colaborativo multicéntrico para el diagnóstico molecular de hemofilia $A$ y $B$ ( $H A, H B$, respectivamente), y el asesoramiento genético a las familias afectadas. El programa colaborativo se desarrolló entre el Grupo de Patología Molecular de la Universidad Nacional de Colombia, el Grupo de Oncohematología Pediátrica de la Fundación HOMI y el Centro de Investigaciones Clínicas de la Clínica Infantil Colsubsidio.

Materiales y métodos: se incluyeron los pacientes con diagnóstico de HA para el año 2015 en los centros de la Fundación HOMI y la Clínica Infantil Colsubsidio, donde se analizaron 33 pacientes. Para HB, se incluyeron 12 pacientes para el año 2017.

Resultados: para la HA, se identificó la inversión 22 en 14/33 (42\%), en 3/33 (9\%) la inversión 1, en 3/33 pacientes (9\%) gran deleción y en 11/33 (33\%) variaciones puntuales o pequeñas deleciones. Para la HB, se han identificado nueve mutaciones únicas: cuatro missense, dos nonsense, dos deleciones grandes $>50$ pb y una mutación en la región UTR 3', que han sido reportadas previamente. Tres pacientes presentan una mutación que afecta el splicing.

Conclusiones: esta alianza permitió identificar las mutaciones responsables de la mayoría de los pacientes con HA y HB, así como brindar un adecuado asesoramiento genético a las familias afectadas, lo cual aporta al abordaje integral. Estos estudios son de gran importancia, ya que contribuyen al entendimiento del comportamiento clínico de estas entidades. 